\title{
Natural history of sleep disordered breathing in prepubertal children transitioning to adolescence
}

\author{
Edward O. Bixler ${ }^{1}$, Julio Fernandez-Mendoza ${ }^{1}$, Duanping Liao ${ }^{2}$, Susan Calhoun ${ }^{1}$, \\ Sol M. Rodriguez-Colon ${ }^{2}$, Jordan Gaines ${ }^{1}$, Fan $\mathrm{He}^{2}$ and Alexandros N. Vgontzas ${ }^{1}$
}

Affiliations:

${ }^{1}$ Dept of Psychiatry, Penn State University, College of Medicine, Hershey, PA, USA.

${ }^{2}$ Dept of Public Health Sciences, Penn State University, College of Medicine, Hershey, PA, USA.

\section{Correspondence:}

Edward O. Bixler, Sleep Research and Treatment Center, Dept of Psychiatry, Penn State University, 500 University Dr., MC: H073, Hershey, PA 17033, USA.

E-mail: ebixlerahmc.psu.edu

ABSTRACT Because there is a lack of agreed upon diagnostic criteria, it is critical to understand the natural history of obstructive sleep apnoea (OSA) in children in order to establish treatment strategies based on objective data.

The Penn State Child Cohort is a representative, general-population sample of 700 elementary school children at baseline, of whom 421 were reassessed 8 years later, during adolescence.

The remission of childhood apnoea-hypopnoea index (AHI) $\geqslant 2$ events per $\mathrm{h}$ in adolescence was $52.9 \%$. Using the higher threshold of AHI $\geqslant 5$ events per h, remission was $100.0 \%$, with $50.0 \%$ partially remitting to AHI $2-<5$ events per $h$ and the other half remitting to AHI $<2$ events per $h$. The incidence of adolescent $\mathrm{AHI} \geqslant 2$ events per $\mathrm{h}$ in those with childhood $\mathrm{AHI}<2$ events per $\mathrm{h}$ was $36.5 \%$, while the incidence of $\mathrm{AHI} \geqslant 5$ events per $\mathrm{h}$ in those with childhood $\mathrm{AHI}<5$ events per $\mathrm{h}$ was $10.6 \%$. This longitudinal study confirms that prepubertal OSA tends to resolve naturally during the transition to adolescence, and that primary snoring and mild sleep disordered breathing (SDB) do not appear to be strongly associated with progression to more severe SDB.

The key risk factors for SDB in adolescence are similar to those found in middle-aged adults (i.e. male sex, older age and obesity). Moreover, consistent with recent studies in adults, this study includes the novel cross-sectional finding that visceral fat is associated with SDB as early as adolescence.

@ERSpublications

Prepubertal sleep disordered breathing tends to resolve naturally during transition to adolescence http://ow.ly/X2Uv8

Editorial comment in: Eur Respir J 2016; 47: 1310-1312.

Received: Oct 262014 | Accepted after revision: Dec 202015 | First published online: Feb 042016

Support Statement: This study was partially supported by US National Institutes of Health grants R01HL063772, R01 HL097165, C06 RR016499 and UL1TR000127. Funding information for this article has been deposited with FundRef.

Conflict of interest: Disclosures can be found alongside the online version of this article at erj.ersjournals.com

Copyright OERS 2016 


\section{Introduction}

The natural history of sleep disordered breathing (SDB) in school-aged prepubertal children is not well understood. Previous natural history studies using polysomnography (PSG) have been largely focused on primary snoring [1-4]. Two of these studies were based on clinical samples $[1,2]$ and two were based on population samples $[3,4]$. These studies reported a strong tendency to remit or persist and a limited tendency to progress to more severe SDB. There have been only two studies that have assessed the natural history of SDB in children without focusing on primary snoring and both of these studies were based on population samples $[5,6]$. A significant problem exists in that there is no consensus for diagnostic criteria for prepubertal or pubertal children, which creates a challenge in establishing these values. The threshold used to estimate SDB in the two studies not focused on primary snoring ranged from respiratory disturbance index $\geqslant 1$ event per $\mathrm{h}[5]$ to apnoea-hypopnoea index (AHI) $\geqslant 5$ events per h or apnoea index $\geqslant 1$ event per $\mathrm{h}$ [6]. Neither of these studies included a wide range of potential risk factors including assessment of visceral obesity. Thus, in order to establish the natural history in adolescents, including incidence, persistence, remission and risk factors, the estimate needs to be based on a PSG assessment and include a wide range of potential risk factors.

The Penn State Child Cohort (PSCC) is a representative population sample of 700 young children (aged 5-12 years) with a reassessment established approximately 8 years after the baseline evaluation during adolescence (12-23 years). A very large set of potential risk factors including demographics, PSG findings, and anatomical and metabolic were factors assessed at baseline and at follow-up. Thus, the purpose of this study was to assess the natural history and potential risk factors for SDB in adolescent children drawn from a representative population sample.

\section{Methods}

Population

The baseline portion of the PSCC has been previously described [7, 8]. In brief, it was designed as a two-phase study with the first phase collecting general information from the parent about their child's sleep and behavioural patterns. In order to establish a representative sample, we assessed all 18 public elementary schools (from kindergarten to fifth grade of the US education system) within three school districts within Dauphin County, PA, USA. We sent a questionnaire home with every child $(\mathrm{n}=7312)$ [9] to be completed by the parent and 5740 were returned for a response rate of $79 \%$ for phase I. Phase II of the baseline study randomly selected 1000 children based on stratification for grade, sex and risk of SDB from the parent's returned questionnaires, and 700 children completed this phase for a response rate of 70\%. Each child spent one night in our Clinical Research Center (CRC) (Penn State University, Hershey, PA, USA) with a parent. During this time, the child also completed a detailed evaluation including a physical and psychometric examination.

In the follow-up, we invited the 700 subjects to return for another visit to the CRC after an average of 8.4 years. Among the 700 subjects, 421 completed the follow-up examination during 2010-2015, yielding a response rate of $60 \%$. The loss to follow-up was mainly due to subjects moving out of the central Pennsylvania area. However, no differences in the baseline demographic characteristics were observed between subjects who participated in the follow-up study examination and those who did not, with only slightly fewer ethnic-minority children (table 1). Based on our protocol, in both assessments, all subjects with an AHI $\geqslant 5$ events per $h$, as well as those with any abnormal values in psychometric screening, physical examination or blood samples, had a report sent to their parents and primary care provider, who

TABLE 1 Baseline characteristics of children who were and were not included in the follow-up study as adolescents

\begin{tabular}{lccc} 
& Not included & Included & p-value \\
\hline Children $\mathbf{n}$ & 279 & 421 & \\
Age years & $9.1 \pm 0.1$ & $9.2 \pm 0.8$ & 0.525 \\
Females \% & 50.2 & 46.1 & 0.288 \\
Ethnic minority \% & 26.5 & 21.9 & 0.155 \\
BMI percentile & $63.0 \pm 1.8$ & $63.3 \pm 1.4$ & 0.894 \\
Waist cm & $65.0 \pm 0.6$ & $65.7 \pm 0.5$ & 0.399 \\
Neck cm & $28.9 \pm 0.3$ & $28.9 \pm 0.2$ & 0.918 \\
AHI events per $\mathbf{h}$ & $0.9 \pm 0.1$ & $0.8 \pm 0.1$ & 0.491 \\
\hline
\end{tabular}

Data are presented as mean \pm SD unless otherwise stated. BMI: body mass index; AHI: apnoea-hypopnoea index. 
recommended any treatment. The study protocol was approved by Penn State University College of Medicine Institutional Review Board. Written informed consent was obtained from participants, and their parents or legal guardians if younger than 18 years.

\section{Sleep laboratory}

Both at baseline and follow-up, the child's sleep was continuously monitored for $9 \mathrm{~h}$ with seven-channel electroencephalography, electro-oculography and electromyography. Respiration was monitored with nasal pressure, a thermocouple, and thoracic and abdominal strain gauges. Snoring sounds were monitored by a microphone attached to the throat and were defined as the presence or absence of any snoring sounds. Haemoglobin oxygen saturation $\left(\mathrm{S}_{\mathrm{PO}_{2}}\right)$ was obtained from the finger. The sleep records were subsequently scored independently according to standardised criteria [10]. An apnoea was defined as a cessation of airflow with a minimum duration of $5 \mathrm{~s}$ for those aged $<16$ years and $10 \mathrm{~s}$ for those aged $\geqslant 16$ years with an associated out-of-phase strain gauge movement. A hypopnea was defined as a reduction of airflow of approximately $50 \%$ with an associated decrease in $\mathrm{SpO}_{2}$ of $\geqslant 3 \%$ or an associated arousal. AHI was calculated as the number of apnoeas and hypopnoeas summed per hour of sleep. Objectively monitored snoring was used in this study. At follow-up, we categorised SDB into four groups: AHI $\geqslant 5$ events per $h$, AHI $2-<5$ events per $\mathrm{h}$ (i.e. an $\mathrm{AHI} \geqslant 2$ and $<5$ events per $\mathrm{h}$ ), primary snoring (i.e. AHI $<2$ events per $\mathrm{h}$ and the presence of snoring detected during the sleep period) and no SDB (i.e. AHI $<2$ events per h without snoring). When assessing risk factors for incident $\mathrm{SDB}$, children with an $\mathrm{AHI} \geqslant 5$ events per $\mathrm{h}$ at baseline were excluded from the analyses and mild levels of SDB at baseline (i.e. baseline snore or AHI $2-<5$ events per $\mathrm{h}$ ) were included as a risk factor for incident SDB.

\section{Key measurements}

We completed a similar evaluation to the baseline assessment, which included a full physical examination, psychometric assessment, parent-reported assessment of their child's medical and sleep history, and spending one night in the sleep laboratory. We expanded the follow-up evaluation by including a Holter monitor of the ECG, seven nights of actigraphic recording of sleep and physical exercise in the home environment, and a whole-body dual-energy X-ray absorptiometry (DXA) scan. Participants were examined in the CRC; after undergoing a DXA scan in the afternoon, a detailed physical examination and psychometric assessment protocol was performed. Participants then stayed overnight in the sleep laboratory to complete a standardised PSG recording. The physical examination was completed in the evening prior to the PSG and included measurements of: height; weight; hip, waist and neck circumference; and blood pressure. At baseline, this examination also included a visual evaluation of the nose and throat by an ear, nose and throat (ENT) specialist and an evaluation of the respiratory function by a paediatric pulmonologist. Blood pressure was measured using an automated system (Vital Signs Monitor; Welch Allyn, Skaneateles Falls, NY, USA). Height was measured in centimetres using a stadiometer (SECA Corp., Hanover, MD, USA) and weight was assessed in kilograms (Cardinal Scale Manufacturing, Webb City, MO, USA); age- and sex-adjusted body mass index (BMI) percentile was calculated based on the formula and data from the 2002 US Centers for Disease Control and Prevention growth charts [11]. The waist was measured in centimetres at the top of the iliac crest and the neck at the cricothyroid membrane. Whole-body DXA scan was used to measure the adipose tissue distribution in abdominal region at the follow-up examination. DXA scan was performed by using Hologic Discovery W scanner (Hologic Inc., Waltham, MA, USA). Subjects were required to remove all metal, plastic and rubber materials to avoid any impact on X-ray beams. Android region (waist), gynoid region (hips), visceral adipose tissue (VAT) and subcutaneous adipose tissue (SAT) were selected as regions of interest (ROIs) to assess abdominal obesity. Detailed ROI defining methods have been described elsewhere [12, 13]. All ROIs were identified by Hologic APEX 4.0 software (Hologic Inc., Bedford, MA, USA) and visually verified by an experienced investigator. Android/gynoid fat mass ratio, android/whole-body fat-mass proportion (AWP), gynoid/whole-body fat-mass proportion (GWP), VAT and SAT areas were used in this report.

\section{Statistical analysis}

Univariate comparisons were made using ANOVA or Chi-squared analysis. In order to establish the relative independent contribution of all potential risk factors, we further analysed the data from a multivariate perspective using multinomial logistic regression with no SDB as the reference. In this study, we report risk factors for incident SDB based on those with $\mathrm{AHI}<5$ events per $\mathrm{h}$ at baseline. In order to further assess these models, we completed another analysis for risk factors excluding those at baseline with an $\mathrm{AHI} \geqslant 2$ events per $\mathrm{h}$ (not reported) and observed the same risk pattern.

The initial model of this analysis included all variables with a univariate association of $\mathrm{p}<0.40$. We assessed the three levels of SDB, each compared to those with no SDB. The initial model included change 
in BMI percentile while controlling for baseline BMI percentile. Further models addressed more precise measures of obesity, including waist and neck circumference and DXA measures. These systematic models enabled us to identify changing patterns of association at various thresholds of severity of SDB. All analyses were performed using SPSS Statistics version 22 (IBM, Armonk, NY, USA).

\section{Results}

Characteristics of the sample

The final sample of 421 adolescents and young adults consisted of $45.8 \%$ girls compared to $47.7 \%$ at baseline. The age range was $12-23$ years, with an average age of $16.9 \pm 2.3$ years; while the age range at baseline was 5-12 years. Approximately one quarter (22.2\%) of our sample was ethnic minority compared to $23.7 \%$ at baseline. Specifically, the ethnic-minority distribution was $12.8 \%$ and $13.7 \%$ for non-Hispanic black and $6.5 \%$ and $6.3 \%$ for Hispanic at follow-up and baseline, respectively. Our sample had an average BMI percentile of $65.3 \pm 1.4$ and $15.2 \%$ were obese. The average AHI was $2.7 \pm 0.3$ events per h of sleep with a maximum value of 91.9 events per $\mathrm{h}$, and an interquartile range of $0.6,1.5$ and 2.9 events per $\mathrm{h}$ of sleep. The prevalence of primary snoring and AHI $2-<5$ events per h was $25.3 \%$, and $27.0 \%$, respectively.

\section{Natural history and incidence}

The remission of $\mathrm{AHI} \geqslant 5$ events per $\mathrm{h}$ was $100 \%$, with half of the subjects (50.0\%) partially remitting into AHI $2-<5$ events per h (table 2). The overall incidence of AHI $\geqslant 5$ events per $\mathrm{h}$ was $10.6 \%$. For those subjects with primary snoring at baseline, the persistence rate was $30.3 \%$, while $31.5 \%$ remitted, and $25.8 \%$ and $12.4 \%$ evolved into AHI $2-<5$ and $\geqslant 5$ events per h, respectively. For subjects with AHI $2-<5$ events per $\mathrm{h}$ at baseline, the persistence rate was $33.3 \%$, while $53.3 \%$ remitted and $13.3 \%$ evolved into AHI $\geqslant 5$ events per $h$.

\section{Risk factors for SDB}

The distribution of demographic factors and potential risk factors for SDB measured at baseline and at follow-up is presented in table 3. Childhood factors that were potentially associated with SDB, defined as $\mathrm{p}<0.40$, included age, sex, ethnic minority, BMI percentile, waist and neck circumference, chronic sinusitis, palate length, and wheeze. In adolescence, age, BMI percentile or change in BMI percentile, minimum $\mathrm{SpO}_{2}$, history of tonsillectomy or adenoidectomy, Tanner stage, chronic sinusitis/rhinitis, and thyroid and respiratory night-time symptoms were potentially associated with SDB.

A multivariate stepwise logistic regression identified the relative contribution of all potential risk factors by assessing the three levels of SDB, each compared to no SDB (table 4). At the lowest threshold (i.e. primary snoring), a marginal association with male sex was observed. Significant risk factors for AHI $2-<5$ and $\geqslant 5$ events per $\mathrm{h}$ were male sex, older age, ethnic minority and greater change in BMI percentile while controlling for baseline BMI percentile. When follow-up BMI percentile replaced change in BMI percentile, similar results were obtained.

\section{Systematic assessment of detailed measures of body fat composition}

Given the strong association of follow-up whole-body obesity measures (i.e. change in BMI percentile or follow-up BMI percentile) with SDB, we systematically assessed the association with more detailed measures of body fat composition. As shown in model 1 of table 5, neck circumference was not associated with any level of SDB, while waist circumference was associated with AHI $\geqslant 5$ events per h (OR 1.89). We

TABLE 2 Persistence, remission and incidence rates of sleep disordered breathing (SDB)

\begin{tabular}{|c|c|c|c|c|c|c|}
\hline & Baseline & \multicolumn{5}{|c|}{ Follow-up } \\
\hline \multirow[t]{3}{*}{ Remission } & Snoring & $28 / 89(31.5)$ & & & & 28/89 (31.5) \\
\hline & AHI $2-<5$ events per $h$ & $16 / 45(35.6)$ & $8 / 45$ (17.8) & & & $24 / 45$ (53.3) \\
\hline & $\mathrm{AHI} \geqslant 5$ events per $\mathrm{h}$ & $1 / 6(16.7)$ & $2 / 6(33.3)$ & $3 / 6(50.0)$ & & $6 / 6(100.0)$ \\
\hline Persistence & & $110 / 281(39.1)$ & $27 / 89$ (30.3) & $15 / 45$ (33.3) & $0 / 6(0.0)$ & $152 / 421(36.1)$ \\
\hline \multirow[t]{2}{*}{ Incidence } & None & & $70 / 281(24.9)$ & $74 / 281$ (26.3) & $27 / 281(9.6)$ & \\
\hline & Overall & & $70 / 281$ (24.9) & $97 / 370(26.2)$ & $44 / 415(10.6)$ & \\
\hline
\end{tabular}

Data are presented as n/N (\%). AHI: apnoea-hypopnoea index. 
TABLE 3 Potential risk factors for incident sleep disordered breathing (SDB) in adolescence

\begin{tabular}{|c|c|c|c|c|c|}
\hline & No SDB & Snoring & AHI $2-<5$ events per $h$ & $A H I \geqslant 5$ events per $h$ & p-value \\
\hline Males \% & 44.2 & 51.4 & 67.0 & 70.5 & $<0.001$ \\
\hline Hispanic & 4.5 & 5.7 & 8.9 & 9.1 & \\
\hline Other & 2.6 & 1.9 & 2.7 & 6.8 & \\
\hline \multicolumn{6}{|l|}{ Baseline } \\
\hline Waist cm & $63.9 \pm 9.7$ & $65.2 \pm 9.6$ & $66.7 \pm 10.7$ & $70.3 \pm 14.1$ & 0.003 \\
\hline Neck cm & $28.2 \pm 0.3$ & $29.2 \pm 0.6$ & $29.2 \pm 0.3$ & $30.4 \pm 0.6$ & 0.017 \\
\hline Tonsil size \% & & & & & 0.945 \\
\hline Tonsil removed & 7.9 & 7.1 & 6.7 & 7.0 & \\
\hline Normal & 52.5 & 57.1 & 53.3 & 45.6 & \\
\hline Abnormal & 39.6 & 35.7 & 40.0 & 46.5 & \\
\hline Allergies \% & 40.1 & 38.1 & 41.8 & 29.5 & 0.544 \\
\hline \multicolumn{6}{|l|}{ Follow-up } \\
\hline Age years & $16.4 \pm 2.1$ & $16.6 \pm 2.2$ & $17.3 \pm 2.2$ & $18.4 \pm 2.1$ & $<0.001$ \\
\hline History of tonsillectomy or adenoidectomy $\%$ & 7.8 & 9.5 & 14.3 & 18.2 & 0.147 \\
\hline Tanner $\%$ & & & & & 0.148 \\
\hline Prepubertal & 0.7 & 1.0 & 1.9 & 0.0 & \\
\hline Early pubertal & 3.4 & 0.0 & 0.0 & 0.0 & \\
\hline Mid puberty & 14.3 & 18.3 & 11.1 & 7.3 & \\
\hline Late puberty & 45.6 & 44.2 & 52.8 & 41.5 & \\
\hline Adulthood & 36.1 & 36.5 & 34.3 & 51.2 & \\
\hline BMI percentile & $60.0 \pm 28.5$ & $61.5 \pm 28.8$ & $70.8 \pm 26.5$ & $79.0 \pm 25.6$ & $<0.001$ \\
\hline Change in BMI percentile \% & $0.1 \pm 22.2$ & $0.2 \pm 23.0$ & $3.8 \pm 23.0$ & $9.0 \pm 24.1$ & 0.090 \\
\hline Minimum $\mathrm{SpO}_{2} \%$ & $92.0 \pm 5.6$ & $91.7 \pm 4.2$ & $91.0 \pm 5.0$ & $90.1 \pm 6.6$ & 0.122 \\
\hline Chronic sinusitis/rhinitis \% & 6.5 & 6.7 & 6.3 & 13.6 & 0.391 \\
\hline Heartburn \% & 12.4 & 12.5 & 17.0 & 18.2 & 0.592 \\
\hline Abdominal pain \% & 9.2 & 7.6 & 9.8 & 2.3 & 0.445 \\
\hline Regurgitation \% & 3.9 & 0.0 & 6.3 & 0.0 & 0.034 \\
\hline Vomiting \% & 2.0 & 2.9 & 2.7 & 0.0 & 0.713 \\
\hline Chronic cough \% & 3.3 & 3.8 & 7.1 & 2.3 & 0.382 \\
\hline Chronic cough with exercise $\%$ & 8.5 & 10.5 & 10.7 & 6.8 & 0.835 \\
\hline Wheezing/dyspnoea \% & 13.1 & 19.0 & 19.6 & 6.8 & 0.131 \\
\hline Respiratory night-time symptoms \% & 2.0 & 3.8 & 5.4 & 6.8 & 0.362 \\
\hline Asthma \% & 5.8 & 11.2 & 8.7 & 4.5 & 0.340 \\
\hline Headaches \% & 26.1 & 25.0 & 23.2 & 31.8 & 0.737 \\
\hline Confusional episodes \% & 1.3 & 1.9 & 2.7 & 0.0 & 0.664 \\
\hline Seizures \% & 3.9 & 3.8 & 1.8 & 0.0 & 0.443 \\
\hline CNS trauma \% & 15.7 & 12.5 & 15.2 & 18.2 & 0.821 \\
\hline Thyroid disease \% & 0.7 & 1.0 & 1.8 & 4.5 & 0.272 \\
\hline Joint pain \% & 13.9 & 21.0 & 17.0 & 11.4 & 0.372 \\
\hline Allergies \% & 60.1 & 57.1 & 62.5 & 36.4 & 0.022 \\
\hline Toxic environmental exposure $\%$ & 3.9 & 1.0 & 3.6 & 4.5 & 0.517 \\
\hline
\end{tabular}

Data are presented as mean \pm SD unless otherwise stated. This table is based on subjects who were included in both the baseline and follow-up assessment excluding those that had an apnoea-hypopnoea index (AHI) $\geqslant 5$ events per $\mathrm{h}$ at baseline. The statistical analysis of the detailed obesity variables was based on normalised values. The descriptive data reported in this table represent the recorded data. These variables include: neck and waist circumference; waist/whole body fat ratio; hip/whole body fat ratio; visceral adipose tissue; and subcutaneous adipose tissue. BMI: body mass index; $\mathrm{SpO}_{2}$ : arterial oxygen saturation measured by pulse oximetry; CNS: central nervous system. 
TABLE 4 Multivariate model of risk factors independently associated with incident sleep disordered breathing in adolescence

\begin{tabular}{lccc} 
& Snoring & AHI $\mathbf{2 - < 5}$ events per $\mathbf{~}$ & AHI $\geqslant \mathbf{5}$ events per $\mathbf{h}$ \\
\hline Age & $1.06(0.91-1.22)$ & $1.31(1.13-1.53)$ & $1.58(1.28-1.97)$ \\
Change in BMI percentile \% & $1.00(0.99-1.01)$ & $1.02(1.01-1.03)$ & $1.05(1.02-1.07)$ \\
BMI percentile \% & $1.00(0.99-1.01)$ & $1.01(1.00-1.03)$ & $1.03(1.01-1.04)$ \\
Ethnic minority & $1.05(0.54-2.07)$ & $2.25(1.18-4.29)$ & $1.61(0.64-4.10)$ \\
Female sex & $0.65(0.37-1.16)$ & $0.28(0.15-0.53)$ & $0.14(0.06-0.36)$ \\
\hline
\end{tabular}

Data are presented as OR $(95 \% \mathrm{CI})$. A multinomial logistic regression model was used to identify those risk factors out of a larger subset of potential risk factors that were independently associated with incident sleep disordered breathing. This model was the result of entering all potential variables with $p<0.40$, excluding those variables assessing body fat composition in detail, and waist and neck circumference. AHI: apnoea-hypopnoea index; BMI: body mass index. " : baseline BMI percentile.

further examined the association of body fat composition at follow-up (i.e. DXA). In model 2, we observed that AWP was significantly associated with AHI $2-<5$ events per h (OR 1.70) and AHI $\geqslant 5$ events per $h$ (OR 1.88) but not primary snoring. In contrast, GWP was not associated with any level of SDB. In model 3, we observed that only VAT was significantly associated with an AHI $\geqslant 5$ events per h (OR 2.78).

\section{Discussion}

This is the first representative, population-based study that has addressed the natural history of SDB, including incidence, persistence, remission and risk factors, in an adolescent sample based on a very large set of potential variables assessed at both baseline and follow-up. The remission of AHI $\geqslant 5$ events per $h$ was $100 \%$, while its incidence peaked to about $10 \%$ during adolescence. Furthermore, for those subjects categorised as primary snorers at baseline, the incidence of AHI $\geqslant 5$ events per $\mathrm{h}$ at follow-up was only $12.4 \%$, which was similar to those without any history of SDB. A Chinese cohort evaluating a 4-year follow-up of children aged 6-13 years with primary snoring reported a slightly lower incidence of AHI $\geqslant 5$ events per $\mathrm{h}$ at follow-up of $7.1 \%$ [4]. Moreover, within our cohort, for those with AHI $2-<5$ events per $\mathrm{h}$ at baseline, we observed a 53.3\% remission at follow-up, with only $13.3 \%$ evolving into $\mathrm{AHI} \geqslant 5$ events per $\mathrm{h}$, suggesting that milder forms of childhood SDB do not appear to be on a continuum with adolescent

TABLE 5 Multivariate models systematically assessing detailed body fat composition

\begin{tabular}{|c|c|c|c|}
\hline & Snoring & AHI $2-<5$ events per $h$ & $A H I \geqslant 5$ events per $h$ \\
\hline \multicolumn{4}{|l|}{ Model 1} \\
\hline Age & $1.03(0.91-1.16)$ & $1.18(1.04-1.34)$ & $1.43(1.20-1.70)$ \\
\hline Female & $0.76(0.41-1.41)$ & $0.46(0.24-0.88)$ & $0.38(0.15-0.94)$ \\
\hline Ethnic minority & $1.08(0.56-2.10)$ & $2.50(1.35-4.64)$ & $1.71(0.69-4.26)$ \\
\hline zNeck & $1.17(0.56-2.42)$ & $1.69(0.84-3.39)$ & $1.50(0.71-3.15)$ \\
\hline zWaist & $1.17(0.75-1.83)$ & $1.24(0.80-1.91)$ & $1.89(1.15-3.10)$ \\
\hline \multicolumn{4}{|l|}{ Model 2} \\
\hline Age & $1.05(0.92-1.19)$ & $1.20(1.05-1.37)$ & $1.43(1.19-1.71)$ \\
\hline Female & $0.61(0.33-1.15)$ & $0.23(0.12-0.45)$ & $0.22(0.09-0.54)$ \\
\hline Ethnic minority & $1.34(0.68-2.64)$ & $3.28(1.71-6.31)$ & $2.15(0.85-5.47)$ \\
\hline zAWP & $1.11(0.83-1.50)$ & $1.70(1.27-2.28)$ & $1.88(1.29-2.74)$ \\
\hline zGWP & $1.13(0.83-1.55)$ & $1.19(0.85-1.67)$ & $1.05(0.65-1.69)$ \\
\hline \multicolumn{4}{|l|}{ Model 3} \\
\hline Age & $1.06(0.94-1.20)$ & $1.25(1.10-1.42)$ & $1.41(1.18-1.67)$ \\
\hline Female & $0.49(0.19-1.27)$ & $0.19(0.07-0.50)$ & $0.34(0.09-1.31)$ \\
\hline Ethnic minority & $1.23(0.62-2.43)$ & $2.91(1.51-5.61)$ & $1.79(0.67-4.75)$ \\
\hline zVAT & $0.83(0.39-1.76)$ & $1.10(0.53-2.28)$ & $2.78(1.11-6.96)$ \\
\hline zSAT & $1.49(0.68-3.30)$ & $1.47(0.67-3.23)$ & $0.66(0.23-1.92)$ \\
\hline \multicolumn{4}{|c|}{$\begin{array}{l}\text { Data are presented as OR }(95 \% \mathrm{CI}) \text {. These multinomial logistic regression models are based on those } \\
\text { variables that were included in preliminary analysis (table } 4) \text { plus a systematic assessment of the detailed } \\
\text { obesity variables. Each of these models includes all variables forced in. AHI: apnoea-hypopnoea index; } \\
\text { z: z-transformed; AWP: android/whole-body fat-mass proportion; GWP: gynoid/whole-body fat-mass } \\
\text { proportion; VAT: visceral adipose tissue; SAT: subcutaneous adipose tissue. }\end{array}$} \\
\hline
\end{tabular}


moderate-to-severe SDB. Finally, beyond the known risk factors for SDB (i.e. male sex, older age and obesity), this study showed, for the first time in a population sample, that visceral fat, as measured by DXA, is associated with SDB as early as adolescence.

We observed a complete remission of $\mathrm{AHI} \geqslant 5$ events per $\mathrm{h}$ over an 8 -year follow-up period. A strong remission rate based on PSG data in prepubertal children has also been reported in the Cleveland Sleep and Health Clinic (CSHC) (Cleveland, OH, USA) [6], in which a remission rate of $91.3 \%$ based on an AHI $\geqslant 5$ events per $\mathrm{h}$ or apnoea index $\geqslant 1$ event per $\mathrm{h}$ after $\sim 8$ years was reported. In the TuCASA (Tucson Children's Assessment of Sleep Apnea) study, a remission rate of $70.8 \%$ was reported based on a lower threshold of RDI $\geqslant 1$ event per $\mathrm{h}$ after 5 years of follow-up [5]. Furthermore, in the recent CHAT (Childhood Adenotonsillectomy Trial) study, a remission of $46 \%$ based on an AHI $\geqslant 2$ event per h in the watchful waiting arm was observed after only 7 months of follow-up [14]. We observed a similar remission rate of $53.3 \%$ for AHI $2-<5$ events per h after an 8 -year follow-up. Consistent with the CSHC findings we observed a trend for "history of tonsillectomy or adenoidectomy" to predict SDB. This latter finding, together with the high remission rates observed, raises concerns regarding early surgical treatment for SDB in prepubertal children, suggesting that prior surgical treatment may not only be ineffective but may also be associated with some risk. In addition, the failure of adenotonsillectomy is also associated with the severity of SDB [14]; thus, guidelines need to be established based on clear clinical outcomes. In fact, the ENT field has previously demonstrated that watchful waiting and adenotonsillectomy had similar long-term outcomes for milder ENT cases [15] and, therefore, they have dramatically reduced the use of adenotonsillectomy [16]. It would appear that a similar strategy would be warranted in the treatment of paediatric SDB, particularly given the role of the key risk factors discussed below. Based on the multivariate analysis of a large set of variables, the significant risk factors for SDB were older age, male sex and obesity. Male sex, ethnic minority and obesity have been previously reported as risk factors for incident SDB within this age group $[7,17,18]$. The data that we collected in this follow-up sample of adolescents also allowed a more detailed assessment of body fat composition. First, we assessed the relative multivariate association of neck and waist circumference. That model indicated that waist circumference was significantly associated with SDB while neck circumference was not. This finding was also reported in our baseline sample [7]. Within the follow-up assessment, we included a full-body DXA evaluation, which allowed us the ability to assess local fat distribution. We first assessed the fat proportion in the waist versus hip areas. The waist area was significantly associated with SDB while the hip area was not. Finally, we assessed subcutaneous versus visceral fat distributions. The final model indicated that only the visceral fat distribution was significantly associated with $\mathrm{AHI} \geqslant 5$ events per $\mathrm{h}$, which is similar to previous studies in clinical and volunteer samples of middle-aged adults $[19,20]$ and adolescents [21-24]; however, these previous studies in adolescents included small samples of prepubertal and pubertal children [21-22], did not account for waist circumference [21-24] and focused on obese subjects [21-24], and only one study included lean controls [24]. Together, these data further support the role of metabolic aberrations in the development and natural progression of SDB as early as adolescence.

Our study has some potential limitations. First, about $60 \%$ of the population-based random sample of the PSCC cohort participated in the follow-up examination, which may introduce a slight selection bias. It remains possible that some of the subjects lost to follow-up completed treatment that we have not accounted for. Nevertheless, no significant differences in demographic characteristics were found between subjects who participated in the follow-up study and those lost to follow-up (table 1), and the PSCC has the best initial baseline response rates among all sleep cohorts in North American children [5, 6]. In addition, the distribution of all levels of baseline SDB was very similar in both samples (snore $20.0 \%$ versus $21.1 \%$, AHI $2-<5$ events per $\mathrm{h} 10.3 \%$ versus $10.7 \%$ and $\mathrm{AHI} \geqslant 5$ events per $\mathrm{h} 1.2 \%$ versus $1.4 \%$ in the baseline and follow-up samples, respectively). Second, the AHI for older adolescents (age $\geqslant 16$ years), the adult scoring criterion (duration $>10 \mathrm{~s}$ ) was used, which would have reduced the incidence estimate. However, this would have strengthened older age as a risk factor in our multivariate models. Thus, our incidence estimates are conservative and we believe that they more closely reflect current clinical practice. Finally, although this study included strong long-term, longitudinal analyses, some associations were based on a cross-sectional analysis of the data (e.g. DXA), where causal inference cannot be drawn.

In conclusion, this longitudinal, population-based study indicates that cases of prepubertal SDB tend to resolve naturally during the transition to adolescence, while the incidence of moderate-to-severe SDB peaks during this developmental period up to $10 \%$. This study also confirms that primary snoring and mild SDB at childhood do not appear to be strongly associated with progression to more severe SDB in adolescence [1-6]. Furthermore, the key risk factors for SDB in adolescence are similar to those found in middle-aged adults (i.e. male sex, older age and obesity). Importantly and consistent with recent studies in adults, this study indicates in a cross-sectional manner that a detailed assessment of body fat composition such as visceral fat is associated with SDB as early as adolescence. 


\section{References}

1 Marcus CL, Hamer A, Loughlin GM. Natural history of primary snoring in children. Pediatr Pulmonol 1998; 26: 6-11.

2 Topol HI, Brooks LJ. Follow-up of primary snoring in children. J Pediatr 2001; 138: 291-293.

3 Anuntaseree W, Kuasirkul S, Suntornlohanakul S. Natural history of snoring and obstructive sleep apnea in Thai school-age children. Ped Pulmonol 2005; 39: 415-420.

4 Li AM, Zhu Y, Au CT, et al. Natural history of primary snoring in school-aged children: a 4-year follow-up study. Chest 2013; 143: 729-735.

5 Goodwin JL, Vasquez MM, Silva GE, et al. Incidence and remission of sleep-disordered breathing and related symptoms in 6- to 17-year old children - the Tucson children's assessment sleep apnea study. J Pediatr 2010; 157: 57-61.

6 Spilsbury JC, Storfer-Isser A, Rosen CL, et al. Remission and incidence of obstructive sleep apnea from middle childhood to late adolescence. Sleep 2015; 38: 23-29.

7 Bixler EO, Vgontzas AN, Lin HM, et al. Sleep disordered breathing in children in a general population sample: prevalence and risk factors. Sleep 2009; 32: 731-736.

8 Bixler EO, Vgontzas AN, Lin H-M, et al. Blood pressure associated with sleep-disordered breathing in a population sample of children. Hypertension 2008; 52: 1-6.

9 Ali NJ, Piston DJ, Stradling JR. Snoring, sleep disturbance and behavior in 4-5 year olds. Arch Dis Child 1993; 68: 360-366.

10 Rechtschaffen A, Kales A. A Manual of Standardized Terminology, Techniques and Scoring System for Sleep Stages of Human Subjects. NIMH Publication 204. Washington, US Government Printing Office, 1968.

11 Kuczmarski RJ, Ogden CL, Guo SS, et al. 2000 CDC Growth Charts for the United States: methods and development. Vital Health Stat 11 2002; 246: 1-190.

12 Hologic Inc. Body Composition User Guide, Document No. MAN-02354 Revision 001. Beford, Hologic Inc., 2010.

13 Kelly TL, Wilson KE, Ruth CR. Estimating visceral fat by dual-energy X-ray absorptiometry. US patent application number US2010-0234719 (Hologic, Inc., 2010).

14 Marcus CL, Moore RH, Rosen CL, et al. A randomized trial of adenotonsillectomy for childhood sleep apnea. N Engl J Med 2013; 368: 2366-2377.

15 Van Staaij BK, Van der Akker EH, Rovers MM, et al. Effectiveness of adenotonsillectomy in children with mild symptoms of throat infections or adenotonsillar hypertrophy: open randomized trial. BMJ 2004; 329: 651-654.

16 Baugh RF, Archer SF, Mitchell RB, et al. Clinical practice guideline: tonsillectomy in children. Otolaryngol Head Neck Surg 2011; 144: S1-30.

17 Rosen CL, Larkin EK, Kirchner HL, et al. Prevalence and risk factors for sleep-disordered breathing in 8- to 11-year-old children: association with race and prematurity. J Pediatr 2003; 142: 383-389.

18 Li AM, So HK, Au CT, et al. Epidemiology of obstructive sleep apnea syndrome in Chinese children: a two phase community study. Thorax 2010; 65: 991-997.

19 Vgontzas AN, Papanicolaou DA, Bixler EO, et al. Sleep apnea and daytime sleepiness and fatigue: relation to visceral obesity, insulin resistance, and hypercytokinemia. J Clin Endocrinol Metab 2000; 85: 1151-1158.

20 Kritikou I, Basta M, Tappouni R, et al. Sleep apnoea and visceral adiposity in middle-aged male and female subjects. Eur Respir J 2013; 41: 601-609.

21 Arens R, Sin S, Nandalike K, et al. Upper airway structure and body fat composition in obese children with obstructive sleep apnea syndrome. Am J Respir Crit Care Med 2011; 183: 782-787.

22 Canapari CA, Hoppin AG, Kinane TB, et al. Relationship between sleep apnea, fat distribution, and insulin resistance in obese children. J Clin Sleep Med 2011; 7: 268-273.

23 Hannon TS, Lee S, Chakravorty S, et al. Sleep-disordered breathing in obese adolescents is associated with visceral adiposity and markers of insulin resistance. Int J Pediatr Obes 2011; 6: 157-160.

24 Yuan H, Schwab RJ, Kim C, et al. Relationship between body fat distribution and upper airway dynamic function during sleep in adolescents. Sleep 2013; 36: 1199-1207. 